\title{
Efficacy of Cetirizine 1\% Versus Minoxidil 5\% Topical Solution in the Treatment of Male Alopecia: A Randomized, Single-blind Controlled Study
}

\author{
Delaram Hossein Mostafa ${ }^{1}$, Aniseh Samadi ${ }^{2}$, Somayeh Niknam³ ${ }^{3}$ Saman Ahmad Nasrollahi ${ }^{2}$, Alexandre Guishard ${ }^{4}$, and \\ Alireza Firooz ${ }^{2}$ \\ ${ }^{1}$ Pharmaceutical sciences branch, Islamic Azad University, Tehran, Iran; ${ }^{2}$ Center for Research \& Training in Skin Diseases \& \\ Leprosy, Tehran University of Medical Sciences, Tehran, Iran.; ${ }^{3}$ Department of Pharmaceutics, Faculty of Pharmacy, Tehran \\ University of Medical Sciences, Tehran, Iran; ${ }^{4}$ Legacy Healthcare SA, Epalinges, Switzerland
}

Corresponding author: Saman Ahmad Nasrollahi PharmD, Ph.D., Center for Research \& Training in Skin Diseases \& Leprosy [CRTSDL], Tehran University of Medical Sciences [TUMS], \#415 Taleqani Ave, Tehran, Iran; TEL: [+9821] 88963804; Fax: [+9821] 88963804; email: snasrollahi@tums.ac.ir

Received, January 24, 2021; Revised, April 12, 2021; Accepted, April 13, 2021; Published, April 23, 2021

\begin{abstract}
Purpose: Prostaglandins play a pivotal role in modulating hair growth cycle. Prostaglandin F2 $\alpha$ and prostaglandin $\mathrm{E}$ have stimulating and prostaglandin $\mathrm{D}$ has inhibitory effects on hair follicle. Cetirizine inhibits release of prostaglandin D2 and stimulates the release of prostaglandin E2. In the present study, the efficacy and safety of twice daily application of topical cetirizine $1 \%$ versus minoxidil $5 \%$ solutions for 16 weeks were compared in male androgenetic alopecia (AGA). Methods: Forty men, aged 18 to 49 years, were randomly divided into two equal groups to apply either cetirizine $1 \%$ or minoxidil $5 \%$ solutions. The study was divided into two phases, a 16-week treatment phase either with cetirizine or minoxidil (anagen phase), followed by an 8-week drug-free (telogen phase) with a follow-up when patients used placebo. Efficacy outcomes included the change in total hair density, vellus and terminal hair density, hair diameter and the percentage of hair in anagen and telogen phases from baseline in 16 and 24 weeks. Results: After 16 weeks, we observed a significant increase in total and vellus hair density in both minoxidil and cetirizine groups, but the improvement was much higher in the minoxidil group. The percentage of hair in the anagen phase also increased in both groups after 16 weeks of treatment, but then diminished after 8 weeks of placebo consumption. No significant adverse reactions associated with the administration of cetirizine solution were reported. Conclusion: Cetirizine 1\% solution was effective in hair growth without any complications for treatment of male AGA.
\end{abstract}

\section{INTRODUCTION}

Androgenetic alopecia (AGA) is the most common cause of scalp hair loss that affects at least $50 \%$ of men by the age of 50 years, and up to $70 \%$ of all males in later life (1). It is characterized by transforming terminal to vellus hair of scalp, corresponding to hair follicle miniaturization during repeated hair cycles with shortened anagen phase (2, $3)$. In susceptible hair follicles, dihydrotestosterone binds to the androgen receptor, and the hormonereceptor complex activates the genes responsible for the gradual transformation of large terminal follicles to minimized follicles (4).

Different prostaglandins play an essential role in the implementation of the hair cycle. Prostaglandins of group F often cause the hair to remain into the anagen phase and hair growth, on the contrary, the prostaglandins of group D, especially prostaglandin $\mathrm{D} 2$, cause the hair to enter into the catagen phase, a resting phase preceeding the telogen phase and then the hair loss (5). Through the adhesion of prostaglandin D2 to its GPR44 receptor, the hair follicle goes to the process resulting in its drop. Some studies have shown androgenetic alopecia to be related to increased prostaglandin D2 (6). A potential way to reduce the amount of prostaglandin D2 is to inhibit prostaglandin D2 synthase, which is responsible for catalysing the isomerisation of prostaglandin $\mathrm{H} 2$ to prostaglandin D2(7).

Cetirizine hydrochloride belongs to a family of medicines called antihistamine which has varied medical uses and is commonly used to relieve allergy symptoms such as watery eyes, runny nose, sneezing, hives, and itching. In addition, it has been demonstrated that cetirizine inhibits release of prostaglandin D2 and stimulates the release of prostaglandin E2 $(6,8)$. Furthermore, it has antiinflammatory properties which can be useful because 
perifollicular micro-inflammation of the scalp is one of deteriorating factors in $\operatorname{AGA}(9,1)$.

Research on the effect of various types of prostaglandins on hair follicle cycling has showed that the $\mathrm{E}$ and $\mathrm{F}$ types (prostaglandin $\mathrm{E}$ and prostaglandin $\mathrm{Fa}$ ) stimulate the hair growth, while the D types (in particular prostaglandin D2) favor the progression of baldness. Indeed, prostaglandin D2 inhibits hair growth, favoring the miniaturization. In this regard, we can state that inhibition of prostaglandin D2 (as di-hydroxytestosterone) through the use of cetirizine will be a new and promising target treatment for AGA. Finally, the lower number of potential side effects, if compared with other drugs commonly used for AGA such as minoxidil, can promote a wider use of cetirizine in the future for the treatment of AGA (10).

In a previous study, topical effect of cetirizine was compared with placebo (10). It seems important to evaluate its effect in comparison with minoxidil since the latter is the standard topical treatment of AGA. The aim of the present study is to assess the efficacy and safety of cetirizine $1 \%$ versus minoxidil $5 \%$ topical solution in men suffering from AGA.

\section{METHODS}

\section{Study Design, Participants, and Interventions}

This research was a randomized, single-blind controlled clinical trial to evaluate the efficacy and safety of topical cetirizine $1 \%$ compared to topical minoxidil 5\% in men with androgenic alopecia (MAGA). This was performed in the Pharmaceutical, Cosmeceutical and Hygienic Evaluation Lab (DermaLab) of the Center of Research \& Training in Skin Diseases \& Leprosy (CRTSDL), Tehran University of Medical Sciences (TUMS), from September 2017 to July 2018. The protocol and informed consent were reviewed and approved by the ethical committee on October 30, 2017 (acceptance code: IR.IAU.PS.REC.1396.136) and was registered in Iranian Registry of Clinical Trial (IRCT) with the registration number: IRCT20161207031288N3. All subjects provided signed informed consent before participating in the trial.

Forty participants were randomly divided into two equal groups to apply $1 \mathrm{~mL}$ of cetirizine $1 \%$ or minoxidil $5 \%$ topically on the balding area twice daily for 16 weeks. Then, from weeks 16 through 24 , both groups received a placebo solution with odor and color similar to active ones.
Subjects were recruited into the study if met the inclusion and exclusion criteria (Table 1) and were blinded to treatment allocation until the study's completion. Moreover, they were asked to maintain the same hair style, color, and length throughout the study.

The hydroalcoholic solution of minoxidil 5\% and cetirizine $1 \%$ with propylene glycol were prepared by a pharmacist and stored in identical bottles, each of them labeled with a container number, dosing instructions, storage conditions, and caution statements.

\section{Assessments}

Primary efficacy endpoint was the change in hair parameters from baseline to week 16. Secondary endpoints included evaluation of hair loss in the last two months after taking placebo (week 24).

\section{Instrumental measurements}

All objective parameters were assessed before and 16 and 24 weeks after intervention by:

Trichoscan assessment, (FotoFinder Systems $\mathrm{GmbH}$, Germany): The trichoscale application of FotoFinder, a new program for documenting and quantifying hair loss, which automatically calculates all the essential parameters of hair growth and hair loss. Parameters measured by this machine include: total hair density, number of vellus hairs, number of terminal hairs, average thickness of hairs, percentage of hair in the anagen and telogen phase.

Manual trichogram test: From 2 regions of the frontal scalp and vertex trichogram, the percentage and frequency of hair in the anagen and telogen phase was investigated and recorded by Dino-lite device (Dino-Lite AM4113ZT Digital Microscope, Taiwan).

\section{Physician Assessment}

The overall condition of the hair was photographically recorded by the standard procedure. Two dermatologists who were blinded to the treatment independently observed those photos and evaluated the degree of changes in comparison to the previous visit in the selected area. The following index was determined: the density of hair has moderately decreased $(-2)$, slightly decreased ($1)$, no change (0), slightly increased $(+1)$, moderately increased $(+2)$.

Table 1. Inclusion and Exclusion Criteria for Recruiting the Participants into the Study 


\begin{tabular}{|c|c|}
\hline \multicolumn{2}{|r|}{ Criteria } \\
\hline Inclusion & $\begin{array}{l}\text { - } \text { Age: } 18 \text { and } 50 \\
\text { - Gender: Male } \\
\text { - } \quad \text { Class of AGA (Hamilton-Norwood } \\
\text { Stages): II to IV (including Type II vertex) }\end{array}$ \\
\hline Exclusion & $\begin{array}{l}\text { - History of allergic reaction to cetirizine, } \\
\text { minoxidil and any other ingredients in the } \\
\text { intervention solution } \\
\text { - Consumption of 5-alpha reductase } \\
\text { inhibitor and isotretinoin over the past } \\
\text { year, as well as nutritional supplements } \\
\text { and herbal remedies for hair growth in the } \\
\text { previous three months } \\
\text { - Chemotherapy in the last year } \\
\text { - Exposure of scalp to radiation in the past } \\
\text { year } \\
\text { - History of uncontrolled blood pressure } \\
\text { and hypotension } \\
\text { Taking systemic steroid for more than } 14 \\
\text { days in the last } 2 \text { months }\end{array}$ \\
\hline
\end{tabular}

\section{Patient Satisfaction}

In each visit, patients' perceptions of their own hair condition in the selected area was inquired. The following index was determined: more worsening hair loss (-2), worsening hair loss (-1), it has not changed (0), improving hair fullness $(+1)$, more improving in hair fullness $(+2)$.

\section{Safety}

The safety of the interventions was attentively followed by asking and seeking the possible signs and symptoms of allergic or skin sensitivity reactions at each visit at week 16 and 24. Dryness, scaling, redness, folliculitis, irritation and itchiness of scalp considered as eventual symptoms were classified as mild, moderate or severe.

\section{Statistical Analyses}

Statistical analysis was performed using the SPSS statistical package (SPSS ${ }^{\circ}$ 20.0; SPSS Inc., Chicago, IL, USA). A paired-sample $\mathrm{T}$ test was performed to detect differences between before and after intervention data in each group and an independent-samples $\mathrm{T}$ Test was used for comparing changes between the two treatment groups. $\mathrm{P}<0.05$ was considered statistically significant.

\section{RESULTS}

Among the 40 men with AGA enrolled into this study (20 men in each group), 30 participants (75\%) completed the study. In the minoxidil group, eight participants left the study (four dropped out after the first visit due to the inconvenience of methods and four dropped out after the second visit for unknown reasons). Whereas in the cetirizine group, two participants dropped out after the first visit. Demographics and baseline characteristics were similar between the treatment groups (Table 2).

\section{Instrumental Measurements}

The comparison between baseline and values taken 16 weeks after consumption of cetirizine or minoxidil solution was considered as study efficacy outcome and the data in the last 8 weeks (after receiving placebo) were obtained to evaluate the continuity of the effect.

Total hair density 16 weeks after treatment significantly increased in both the cetirizine and minoxidil groups, but this enhancement was much more in minoxidil group and there was significant difference between cetirizine and minoxidil (Figure 1A). Also, significant increase in vellus hair density was observed after 16 weeks (Figure 1B). A slight increase in terminal hair density was observed after 16 weeks, but it was not statistically significant (Figure 1C). Enhancement of total and vellus hair density against baseline was observed after 24 weeks in both cetirizine and minoxidil, but there was some reduction in these hair parameters in the minoxidil group against 16 weeks, which was not statistically significant. This reduction was not seen in cetirizine group.

There was no statistical changes in hair diameter after 16 and 24 weeks in any group (data not shown). According to the manual trichogram method, percentage of hair in the anagen phase in both cetirizine and minoxidil groups increased 16 weeks after treatment, while a slight reduction was observed at week 24. However, these changes were not statistically significant (Figure 1D). The pattern of changes in the telogen phase in the cetirizine group was different from the minoxidil group (Figure 1E). In the minoxidil group, a slight decrease was detected after 16 and 24 weeks, but in the cetirizine group first there was a sharp reduction in percentage of hair in the telogen phase at week 16, then it increased at week 24.

\section{Physician Assessment}

The physicians' opinions about the hair density were perused after 16 and 24 weeks of intervention (Figures 2A, 2B and 3). Based on physicians' opinions, after 16 weeks of treatment in the cetirizine group, hair density in $33 \%$ of participants slightly increased, $50 \%$ of participants did not differ in hair 
density and in $17 \%$ of the subjects hair density slightly decreased. Whereas in the minoxidil group, $25 \%$ had a slight increase in hair density and in $75 \%$ of participants no changes from baseline were detectable (Figure 2A).
In the cetirizine group, after 8 weeks of using the placebo solution, by comparing weeks 16 and 24, hair density in $25 \%$ of participants slightly increased, $41 \%$ experienced no difference in their hair density
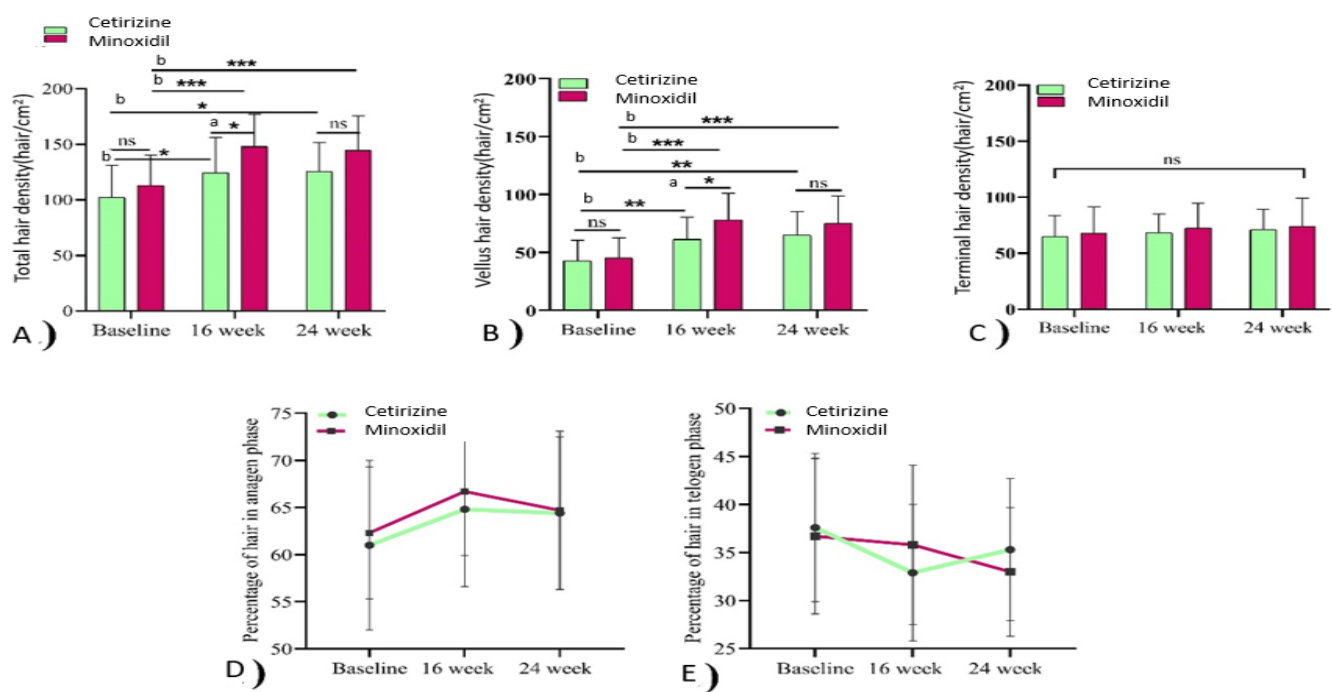

Figure 1. Changes in Biometric Indices of Hair along 24 Weeks of Study in Both Groups Cetirizine, Minoxidil. A) Total hair density, B) vellus hair density, C) terminal hair density, D) Percentage of hair in anagen phase, E) Percentage of hair in telogen phase; a: There was significant difference between cetirizine and minoxidil groups in a specific time $(0,16$ and 24 weeks) b: There was significant difference intra-group (cetirizine or minoxidil) in 16 and 24 week(s) in comparison with baseline. Significant level: $*=$ p-value $<0.05 * *=$ p- value $<0.01 * * *=$ p- value $<0.001$

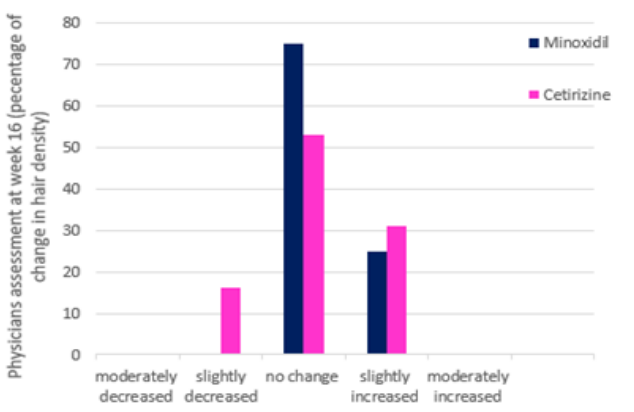

(A)

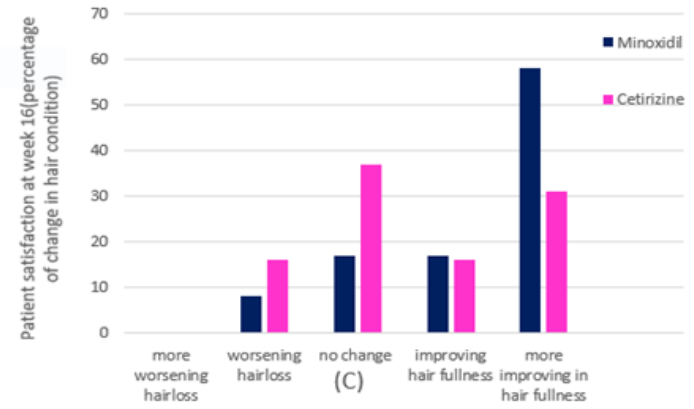

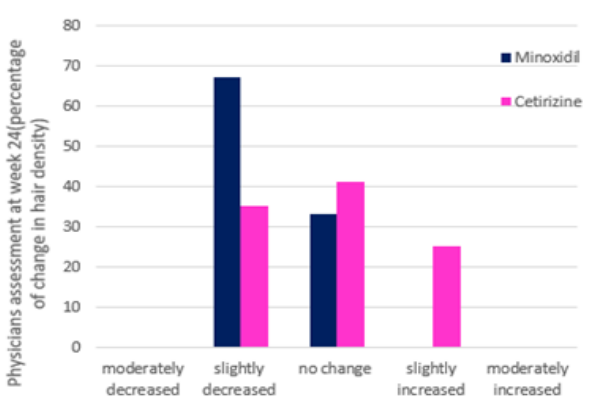

(B)

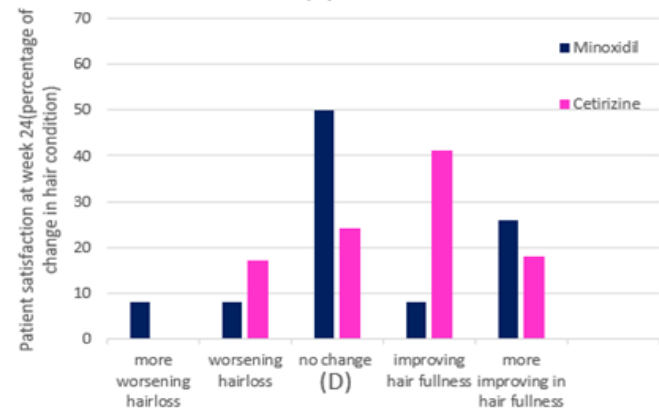

Figure 2. Five Point Rating Scale Used by Physicians and Patients in Both Groups Cetirizine, Minoxidil A) Physicians' assessment after 16 weeks of treatment against baseline, B) Physicians' assessment after 8 weeks of using the placebo solution against week 16, C) Patients' satisfaction after 16 weeks of treatment against baseline, D) Patients' satisfaction after 8 weeks of using the placebo solution against week 16 


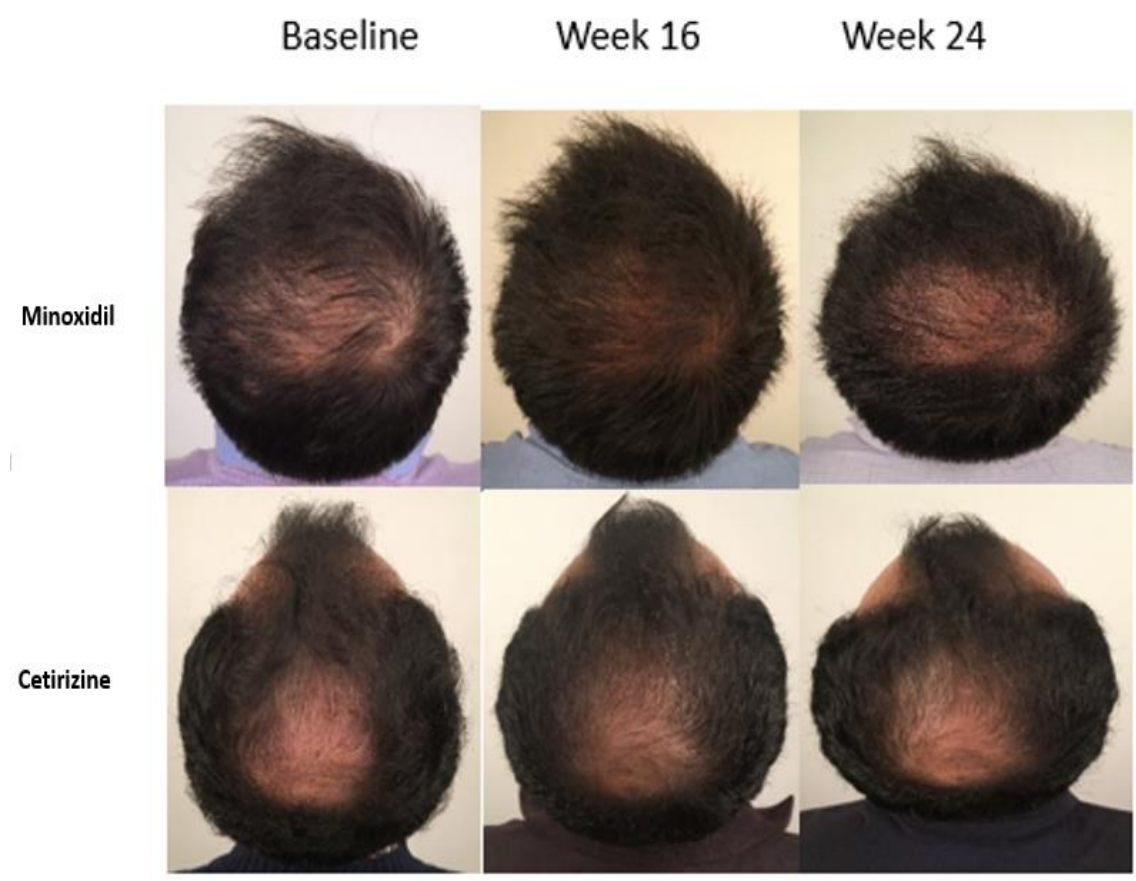

Figure 3. Changes in Hair Density of Case Number 32 and 34 in Patients Treated with Topical Solution of cetirizine $1 \%$ and minoxidil 5\%, respectively, at Baseline, Week 16 and Week 24.

and $34 \%$ had a slight decrease in hair density. Whereas in the minoxidil group, $33 \%$ of the subjects had no change in hair density compared to week 16 , and $67 \%$ of participants had a slight decrease in hair density (Figure 2B).

\section{Patient Satisfaction}

The patients' sensorial evaluation about the condition of their own hair were perused at weeks 16 and 24 of the study (Figure $2 \mathrm{C}$ and D).

In the cetirizine group, after 16 weeks of treatment in comparison to baseline, $31 \%$ of participants reported more improvement in hair fullness, $16 \%$ reported improving hair fullness and $16 \%$ mentioned worsening hair loss. Whereas, $37 \%$ didn't feel any changes in their hair condition. The participants in the minoxidil group expressed more satisfaction about hair growth; $58 \%$ of them reported more improvement in hair fullness, $17 \%$ reported improving hair fullness. Finally, 17\% didn't feel any changes in their hair condition and $8 \%$ declared worsening hair loss.

After 8 weeks of using the placebo solution in comparison to week $16,18 \%$ of participants in the cetirizine group reported more improvement in hair fullness, $41 \%$ felt they had improving hair fullness,
$24 \%$ didn't feel any changes and $17 \%$ expressed worsening hair loss. Whereas in minoxidil group, $26 \%$ reported more improvement in hair fullness, $8 \%$ reported improving hair fullness, $50 \%$ declared no change, and $8 \%$ reported more worsening hair loss.

\section{Safety}

Mild Pruritus (15\%) and scaling (10\%) were the main complaints in the minoxidil group, whereas no side effects were seen in the cetirizine group.

\section{DISCUSSION}

Hair follicle cycle is a sophisticated process with the involvement of many biochemicals and hormones. Organized transition of a hair follicle from anagen (growth phase) to telogen (resting phase) is in the response of molecular signals.

It has been illustrated that prostaglandins have an important impact as modulators in hair cycle. Recently, various studies showed the beneficial effects of prostaglandin F2 $\alpha$ analogues (latanoprost, isopropyl unoprostone and bimatoprost) and prostaglandin E2 analogue (viprostol) on hair growth (11-13). One of the suggested mechanisms for 
minoxidil, as the most popular and the only FDA approved topical agent for treatment of hair loss, is enhancement of prostaglandin endoperoxide synthase-1 activity, which normalizes prostaglandin E2 levels (14).

In contrast, prostaglandin D2 has a negative effect which inhibits hair growth. It seems there exists a counterbalance between the opposing functions of prostaglandin E2 and prostaglandin D2 that controls hair growth in mouse and human skin. Prostaglandin D2, with overexpressing prostaglandin-endoperoxide synthase-2, indirectly induces apoptotic catagen phase and diminish hair lengthening (15). Moreover, there was the hypothesis that sebaceous hyperplasia, which was frequently seen in AGA, is related to the increase in the levels of prostaglandin D2 (5).

Table 2. Participant Demographics and Baseline Characteristics

\begin{tabular}{|c|c|c|}
\hline Characteristic & $\begin{array}{l}\text { Cetirizine group }(N=18) \\
(\text { Mean } \pm \text { SD) }\end{array}$ & $\begin{array}{l}\text { Minoxidil group }(N=12) \\
(\text { Mean } \pm \text { SD) }\end{array}$ \\
\hline Age (years) & $34.5 \pm 7$ & $33.2 \pm 8.4$ \\
\hline Duration of MPHL (years) & $6.2 \pm 4.6$ & $5.2 \pm 3.9$ \\
\hline Positive Family history of AGA & 12 & 10 \\
\hline YES & 12 & 10 \\
\hline NO & 6 & 2 \\
\hline \multicolumn{3}{|l|}{ Hamilton- Norwood Stages } \\
\hline II & 6 & 5 \\
\hline III & 8 & 3 \\
\hline IV & 4 & 4 \\
\hline Total hair density (hair $/ \mathrm{cm}^{2}$ ) & $102.3 \pm 29.1$ & $113 \pm 27.4$ \\
\hline Vellus hair density $\left(\right.$ hair $\left./ \mathrm{cm}^{2}\right)$ & $42.9 \pm 17.7$ & $45.3 \pm 17.3$ \\
\hline Terminal hair density (hair/cm²) & $64.7 \pm 18.9$ & $67.9 \pm 23.5$ \\
\hline Hair diameter $(\mu \mathrm{m})$ & $44.8 \pm 9.8$ & $42.7 \pm 21.3$ \\
\hline Percentage of hair in anagen phase & $61 \pm 9$ & $62.3 \pm 7$ \\
\hline Percentage of hair in telogen phase & $37.6 \pm 7.7$ & $36.7 \pm 8.1$ \\
\hline
\end{tabular}

Inhibiting prostaglandin D2 may prevent miniaturization, which demonstrated some positive effect of cetirizine on hair density.

Cetirizine is a potent second-generation H1receptor antagonist which has an inhibitory effect on the release of mast cell mediators like prostaglandin D2 (16) and increases the release of prostaglandin E1 from human monocytes (17).

Hydrochloride salt of cetirizine is a common form of systemic active agent, which is water soluble. Because of the hydrophilic moiety of the hair shaft related to keratin structure, hydrophilic agents like cetirizine hydrochloride can penetrate the dermal papillary directly with the hair follicular shunt. This property makes the cetirizine a good candidate for preparing a topical dosage form.

There were just a few preliminary studies which evaluated the topical solution effect of cetirizine in AGA. The study was conducted on 85 patients from both sex (male and female) which reported the efficacy of cetirizine (as a 1\% solution) versus placebo (base solution) during a 6-month trial. Measurements of terminal and vellus hair counts and diameter, as well as global photography, showed a modest increase in total and terminal hair density and diameter; also a decrease in vellus hair density (10). Another study was a short report of the reformative effect of topical cetirizine in combination with oral vitamin $\mathrm{D}$ in congenital hypotrichosis caused by ectodermal dysplasia. The vellus hair replacement by terminal hair was noticed in this report. Furthermore, after 6 months of treatment, the density of hair on the scalp increased in all patients from clinical and trichoscopic points of view (8). Also, recently published a research article in the journal of cosmetic dermatology which indicated a potential role of cetirizine $1 \%$ in treating $\mathrm{AGA}(18)$.

The present study was a before-after evaluation of cetirizine $1 \%$ solution effect on hair density (total, vellus and terminal), hair diameter and the presence of hair in anagen and telogen phase in comparison with minoxidil $5 \%$ solution as a standard topical treatment of AGA.

After 16 weeks of intervention, there was a significant difference in total and vellus hair density before and after treatment in both the minoxidil and cetirizine groups, but the improvement in hair density (total and vellus) was much higher in the 
minoxidil group. Increase in total hair density subsequently depends on the increase in vellus hair count. This finding supported the idea that minoxidil at the first stage promotes vellus hair production and those vellus hairs turn to terminal hairs with continuing the treatment and it may be indicated that the increase of total hair density is related to growth of vellus hair rather than terminal hair. The next 8 weeks was designed to evaluate the consistency of the therapeutic effect of each intervention by receiving a placebo in both groups. Some reduction in total and vellus hair density was detectable at week 24 compared to week 16 in both groups, but these hair parameters were still significantly higher at week 24 compared to the baseline of both groups which insists on the fact that cetirizine has a better long term profile (remanence) than minoxidil.

Average hair diameter increased in the minoxidil group during 16 weeks of treatment, but diminished by receiving a placebo for 8 weeks. In contrast, both decreases and increases were seen in the cetirizine group at weeks 16 and 24. These changes were not statistically significant in both groups (data not shown), meaning that cetirizine is "as good as" minoxidil in increasing or stabilizing the diameter.

As demonstrated in figure 1, the percentage of hair in anagen phase increased in both groups after 16 weeks and then diminished during 8 weeks of placebo intervention. The pattern of changes in the percentage of hair in telogen phase was different between the cetirizine and minoxidil groups. Primary decrease and secondary increase in the percentage of hair in telogen phase in the cetirizine group were seen. Conversely, continuous reduction in percentage of hair in telogen phase was seen in minoxidil group. There was overall agreement in physicians' assessments and patients' self-evaluations about hair fullness conditions after 16 and 24 weeks of intervention in both groups. For minoxidil, better scores after 16 weeks and more drop in assessment after discontinuing the treatment (between 16 and 24 weeks) were detectable according to subjective evaluations. As previous studies showed, discontinuation of minoxidil does indeed results in the loss of recruited hairs during 1 to 3 months after discontinuing the drug (19).

In the cetirizine group, according to physicians' assessments after 24 weeks, $25 \%$ of patients had slightly increased hair density and based on patient satisfaction, $41 \%$ of patients had improved hair fullness. This result may indicate some dilatory effect for cetirizine in hair growth promotion.

In addition to the beneficial inhibitory effect of cetirizine in prostaglandin D2, by possessing antiinflammatory and anti-allergic properties, it has the least adverse effect compared to minoxidil (Mild Pruritus and scaling). Topical minoxidil may be systematically absorbed and can affect heart function (20). After more than a decade of use, topical minoxidil solution has proven to be a safe and effective treatment for androgenetic alopecia. However, some patients present complaints of pruritus and scaling of the scalp (21). Therefore, cetirizine would be useful for long term use for people contra-indicated or allergic to minoxidil.

According to the results of hair loss after cessation of use in the cetirizine group, it may be concluded that cetirizine can be used as maintenance treatment with fewer side effects. Therefore it can be a new treatment for androgenetic hair loss as well as the common and well established AGA treatments like minoxidil (often a cause of hypertrichosis, contact allergic dermatitis, headache and hypotension).

The major limitation of this research was the low sample size of the study and drops in the number of participants. For approving the cetirizine solution beneficent effects on hair growth, it is essential to reassess these results by similar investigations with a bigger number of participants. Also, based on the outcomes, the duration of drug administration (16 weeks) may not be enough to observe significant changes in all biometric characteristics of the hair. Therefore, further studies with longer durations of treatment and with a non-inferiority design are suggested. Finally, another suggestion is increasing the cetirizine concentration in topical solution.

In this study the concentration of cetirizine solution used was $1 \%$, based on the primary study (10), but there was no information about effective anti-hair loss concentration. Similar to minoxidil (22), however, it is the beneficial effects of cetirizine may be dose-dependent, thus, greater doses may demonstrate improved efficacy. Therefore, it is timely to explore the optimum concentration in further preclinical studies. Moreover, it is interesting to study the combination of cetirizine and minoxidil.

\section{CONCLUSION}

The present study, as a precedential before-after evaluation, found some beneficial effects of topical 
cetirizine in AGA treatment. Because of high safety profile of cetirizine in topical usage, it seems that it will be a useful idea to consider cetirizine as a potential treatment for AGA for more evaluations.

ACKNOWLEDGEMENT. We would like to acknowledge Ms. Samaneh Haghani, and Ms. Sommayeh Yadangi for their technical contributions to this research.

CONFLICT OF INTEREST. None of the authors declare conflict of interest.

\section{REFERENCES}

1. Trüeb RM. Molecular mechanisms of androgenetic alopecia. Experimental Gerontology 2002; 37(8-9): 981-990. DOI: 10.1016/S0531-5565(02)00093-1

2. Kelly Y, Tosti A. Androgenetic Alopecia: Clinical Treatment. InHair and Scalp Treatments 2020 (pp. 91-108). Springer, Cham. DOI: 10.1007/978-3-03021555-2_8

3. York K, Meah N, Bhoyrul B, et al. Treatment review for male pattern hair-loss. Expert Opinion on Pharmacotherapy. 2020 Feb 17:1-0. DOI: 10.1080/14656566.2020.1721463

4. Inui S, Itami S. Molecular basis of androgenetic alopecia: From androgen to paracrine mediators through dermal papilla. Journal of dermatological science. 2011;61(1):1-6. DOI: 10.1016/j.jdermsci.2010.10.015

5. Garza LA, Liu Y, Yang Z, et al. Prostaglandin D2 inhibits hair growth and is elevated in bald scalp of men with androgenetic alopecia. Science translational medicine. 2012;4(126):126ra34-ra34.

DOI: $10.1126 /$ scitranslmed.3003122

6. Patel B, Velasco MA, Gutierrez FT, et al. Addressing androgenetic Alopecia-A complex disorder-with a multilateral treatment strategy. MOJ Bioequiv Availab. 2017;3(1):00025. DOI: 10.15406/mojbb.2017.03.00025

7. Fong $\mathrm{P}$, Tong $\mathrm{HH}, \mathrm{Ng} \mathrm{KH}$, et al. In silico prediction of prostaglandin D2 synthase inhibitors from herbal constituents for the treatment of hair loss. Journal of ethnopharmacology. 2015 Dec 4;175:470-80. DOI: 10.1016/j.jep.2015.10.005

8. Rossi A, Miraglia E, Fortuna M, et al. Topical cetirizine and oral vitamin D: a valid treatment for hypotrichosis caused by ectodermal dysplasia. Journal of the European Academy of Dermatology and Venereology. 2017;31(2):367-70. DOI: 10.1111/jdv.13864

9. Simons FER. Comparative pharmacology of $\mathrm{H} 1$ antihistamines: clinical relevance. The American journal of medicine. 2002;113(9):38-46. DOI: 10.1016/S0002-9343(02)01436-5
10. Rossi A, Campo D, Fortuna M, et al. A preliminary study on topical cetirizine in the therapeutic management of androgenetic alopecia. Journal of Dermatological Treatment. 2018;29(2):149-51. DOI: $10.1080 / 09546634.2017 .1341610$

11. Blume-Peytavi U, Lönnfors S, Hillmann K, et al. A randomized double-blind placebo-controlled pilot study to assess the efficacy of a 24-week topical treatment by latanoprost $0.1 \%$ on hair growth and pigmentation in healthy volunteers with androgenetic alopecia. Journal of the American Academy of Dermatology. 2012;66(5):794-800.

DOI: $10.1016 /$ j.jaad.2011.05.026

12. Zaher H, Gawdat H. Bimatoprost: a potential therapeutic tool for alopecia areata of the scalp. Journal of the Egyptian Women's Dermatologic Society. 2014;11(2):123-7.

DOI: 10.1097/01.EWX.0000444330.33581.2f

13. Sasaki S, Hozumi Y, Kondo S. Influence of prostaglandin F2 $\alpha$ and its analogues on hair regrowth and follicular melanogenesis in a murine model. Experimental dermatology. 2005;14(5):323-8. DOI: 10.1111/j.0906-6705.2005.00270.x

14. Michelet J-F, Commo S, Billoni N, et al. Activation of cytoprotective prostaglandin synthase-1 by minoxidil as a possible explanation for its hair growth-stimulating effect. Journal of investigative dermatology. 1997;108(2):205-9. DOI: 10.1111/1523-1747.ep12334249

15. Müller-Decker K, Leder C, Neumann M, et al. Expression of cyclooxygenase isozymes during morphogenesis and cycling of pelage hair follicles in mouse skin: precocious onset of the first catagen phase and alopecia upon cyclooxygenase-2 overexpression. Journal of investigative dermatology. 2003;121(4):661-8. DOI: 10.1046/j.1523-1747.2003.12473.x

16. Okayama Y, Benyon R, Lowman M, et al. In vitro effects of H1-antihistamines on histamine and PGD2 release from mast cells of human lung, tonsil, and skin. Allergy. 1994;49(4):246-53. DOI: 10.1111/j.1398-9995.1994.tb02657.x

17. Roch-Arveiller M, Tissot M, Idohou N, et al. In vitro effect of cetirizine on PGE 2 release by rat peritoneal macrophages and human monocytes. Agents and actions. $\quad 1994 ; 43(1-2): 13-6 . \quad$ DOI: 10.1007/BF02005756

18. Mohamed S. Zaky, Hassan Abo Khodeir, HebatAllah Ahmed, Mohamed L. Elsaie. Therapeutic implications of topical cetirizine $1 \%$ in treatment of male androgenetic alopecia: A case-controlled study. Journal of Cosmetic Dermatology. 2021;20(4): 1154-1159. DOI: $10.1111 /$ jocd.13940

19. Rogers NE, Avram MR. Medical treatments for male and female pattern hair loss. Journal of the American Academy of Dermatology. 2008 Oct 1;59(4):547-66. DOI: 10.1016/j.jaad.2008.07.001 
20. Georgala S, Befon A, Maniatopoulou E, et al. Topical use of minoxidil in children and systemic side effects. Dermatology. 2006 Dec 1;214(1):101. DOI: $10.1159 / 000096924$

21. Friedman ES, Friedman PM, Cohen DE, et al. Allergic contact dermatitis to topical minoxidil solution: etiology and treatment. Journal of the American Academy of Dermatology. 2002 Feb 1;46(2):309-12.

DOI: $10.1067 / \mathrm{mjd} .2002 .119104$

22. Olsen EA, Dunlap FE, Funicella T, et al. A randomized clinical trial of 5\% topical minoxidil versus $2 \%$ topical minoxidil and placebo in the treatment of androgenetic alopecia in men. Journal of the American Academy of Dermatology. 2002;47(3):377-85. DOI: 10.1067/mjd.2002.124088 